\title{
Vector Transition Classes Generation from Fuzzy Overlapping Classes
}

\author{
Enguerran Grandchamp ${ }^{1}$, Sébastien Régis ${ }^{1}$, and Alain Rousteau ${ }^{2}$ \\ ${ }^{1}$ LAMIA Laboratory, French West Indies University, Guadeloupe, France \\ ${ }^{2}$ DUNECAR Laboratory, French West Indies University, Guadeloupe, France \\ \{egrandch, sregis, aroustea\}@univ-ag.fr
}

\begin{abstract}
We present in this paper a way to create transition classes and to represent them with vector structures. These classes are obtained using a supervised classification algorithm based on fuzzy decision trees. This method is useful to classify data which have a space evolution following a gradient such as forest, where transitions are spread over hundreds of meter, or other natural phenomenon. The vector representation is well adapted for integration in Geographical Information Systems because it is a more flexible structure than the raster representation. The method detailed takes into account local environmental conditions and leads to non regular gradient and fuzzy structures. It allows adding classes, called transition classes, when transition areas are too spread instead of fixing an arbitrary border between classes.
\end{abstract}

Keywords: GIS, decision tree, fuzzy, classification.

\section{Introduction}

Geographical Information Systems (GIS), and their applications, followed a spectacular increase during the last years. Data modeled with these systems are based on geographical positions which could represent simple points or very complex structures such as cities or natural phenomenon (hurricane, rain), networks (water, electric).

Each GIS tool allows manipulating raster data (such as satellite images or results of spatial analysis) or vector data (series of points, lines or polygons).

Since 1997, in the conclusions of the special edition Spatial Data Types for Database Systems 18, the authors underline the importance of data structures in GIS and their lack of adaptation to fuzzy data. Even if some ways have been investigated, the implementation and modeling difficulties as much as computation complexity lead, more than 10 years later, to a very shy advance in this field.

In 1986, Peter Burrough has done the first work concerning fuzzy 2D modeling [3]. Most of the studies on representation and manipulation of 2D fuzzy data use a raster representation ([13], [15], [20], [21]). This representation is based on a regular square partition of the space. The main drawbacks of such models have often been underlined in the literature ([1], [7], [8], [12], [18]). They are linked to (i) a lack of flexibility (ii) a high computation time (iii) a large size of the data (iv) the degradation of the precision linked to the approximation of the rasterization. 
A vector representation uses simple geometric models (points, lines, polygons) to represent the scenes. It is the most adapted structure for GIS and could receive contextual information (semantic) [13]. The main drawback for fuzzy representation is that they are based on strict structures. Only rare studies introduce a vector dimension to fuzzy geo-processing ([2], [17]). They propose modeling fuzzy set with series of regular buffers around the classes.

In this paper, we propose a fuzzy vector model based on non regular structures allowing a more reliable representation of the data. Secondly we propose a strict representation of a set of fuzzy classes by introducing transition classes instead of fixing an arbitrary border to split the space. Indeed, we present a method to create transition classes from a supervised classification based on fuzzy decision trees. This method is particularly useful for the strict classification of data having fuzzy borders. This is the case of the studied forest, where transitions are spread over hundreds of meters and depend on local environmental conditions.

Section 2 presents the modeling with fuzzy classes using decision trees and also gives raw and simplified representations of the classes. Section 3 presents the algorithm to generate the transition classes. Section 4 gives illustrations of the two previous sections and define the reference data. Section 5 gives some perspectives to this work and section 6 is the conclusion.

\section{$2 \quad$ Fuzzy Vector Classes Modeling}

Let $V=\left\{V_{i}, i \in\{1, \ldots, M\}\right\}$ be the vector objects obtained by the union of $N$ information layers $\left(L=\left\{L_{l}, \ldots, L_{N}\right\}\right) . V$ is considered as a partition of the space [10], [11]. Each $V_{i}$ represents a uniform area regarding the information coming from the layers and is then associated to an attribute vector $F_{i}=\left\{F_{i 1}, F_{i 2}, \ldots, F_{i N}\right\}$. Figure 1, illustrates the building of $V$ from two layers.

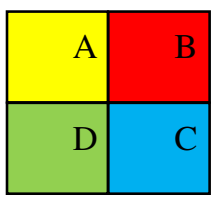

Layer $L_{l}$

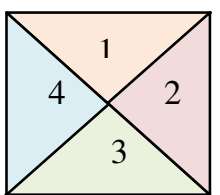

Layer $L_{2}$

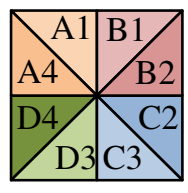

Union

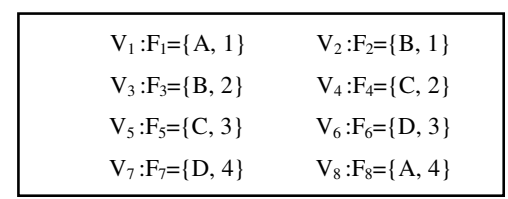

$V$ and Attributes

Fig. 1. Layers union

We consider now the fuzzy classification of the $V_{i}$ using $F_{i}$. The classes are obtained using fuzzy supervised classification methods. We use decision trees such as Functional Trees (FT, [7]) and C4.5 trees [14].

We consider a set of $n$ classes $\left(C_{k}, k \in\{1 \ldots n\}\right)$ and a set $A \subset V$ of labelized $V_{i}$ for learning and cross validation steps.

After the learning step, the decision trees used give for each $V_{i}$ a vector $\mu_{i}=\left\{\mu_{i 1}\right.$, $\left.\ldots, \mu_{i n}\right\}$ which indicates the trust degree of the affectation of the $V_{i}$ to each class $\left(\mu_{i k}\right.$ $=$ trust degree in the assertion $V_{i}$ belongs to class $k$ ). The trust degrees are computed with a logistic regression [9] giving the membership probability of a sample to each 
class. The regression function used is defined as a linear combination of the attributes used during the learning step.

The heart of the class $C_{k}$ is defined by $: H_{k}=\left\{V_{i} \mid i \in\{1 \ldots M\}, \mu_{i k}=1\right\}$

The interior of the class $C_{k}$ is defined by : $E_{k}=\left\{V_{i} \mid i \in\{1 \ldots M\}, \mu_{i k}>0\right\}$

The exterior of the class $C_{k}$ is defined by : $\overline{E_{k}}=\left\{V_{i} \mid i \in\{1 \ldots M\}, \mu_{i k}=0\right\}$

So each class $C_{k}$ is a fuzzy vector map based on the values of the trust degrees of the $V_{i}$ restricted to the class: $m_{k}=\left\{\mu_{l k}, \ldots, \mu_{M k}\right\}$. The display of this kind of map is classic and the grey level depends on the trust degree ( 0 for black and 1 for white).

This is a vector representation because each $V_{i}$ is a vector object. Even if this kind of structure is a reliable internal representation it represents a lot of data (due to the continuous aspect of the membership function) and it could be simplified for more visibility. To solve this problem we propose different simplified views by building groups of spatially close $V_{i}$ having close trust degrees.

The parameters defining a view $v$ are the number of groups $g_{v}$ and the limits of the intervals of the trust degrees $I^{v}=\left\{I_{0}^{v}, I_{2}^{v}, \ldots, I_{g_{v}}^{v}\right\}$ with $I_{0}^{v}=0, I_{g_{v}}^{v}=1$ and $I_{p}^{v}<I_{p+1}^{v}$.

The $p^{\text {th }}$ group $\left(p \in\left\{1 \ldots g_{v}\right\}\right)$ of the class $C_{k} \quad\left(\mathrm{k} \in\left\{\begin{array}{lll}1 & \ldots & n\end{array}\right\}\right)$ regarding the view $v$ is defined by

$$
G_{k p}^{v}=\left\{V_{i} \mid I_{p-1}^{v}<\mu_{i k} \leq I_{p}^{v}\right\}
$$

Adjacent $V_{i}$ belonging to the same group are joined to form a unique vector object.

With such notations

$$
\forall v, E_{k}=\bigcup_{p=1}^{g_{v}} G_{k p}^{v}, \quad \mathrm{~V}=\bigcup_{p=1}^{g_{v}} G_{k p}^{v} \cup \overline{E_{k}} \quad \text { and } \quad H_{k} \subseteq G_{k g_{v}}^{v}
$$

The choice of the limits allows underlying different aspects of the class. Different ways to fix the limits have been identified:

1. Regular partition of $[0,1]$. In this case $I_{p}^{v}=\frac{p}{g_{v}}$

2. Intervals centered on most represented values of the trust degrees. $I_{p}^{v}$ is fixed in order to have $\left|G_{k p}^{v}\right| \simeq \frac{M}{g_{v}}$ (I.I returns the cardinal and $M$ is the number of $V_{i}$, $\left.M=\sum_{p=1}^{g_{v}}\left|G_{k p}^{v}\right|\right)$. In this case we choose the intervals in order to minimize $\sum_{p=1}^{g_{v}}\left(\left|G_{k p}^{v}\right|-\frac{M}{g_{v}}\right)^{2}$

3. Intervals centered on the highest values of the trust degrees. In this case we have to respect the following rule $I_{p+1}^{v}-I_{p}^{v}<I_{p}^{v}-I_{p-1}^{v}$. Every function $f$ which is strictly increasing and concave from $[0,1]$ to $[0,1]$ (with $f(0)=0$ et $f(1)=1$ ) could be used from a regular partition : $I_{p}^{v}=f\left(\frac{p}{g_{v}}\right)$.

\section{Transition Class Generation}

We are now interested on the research of extended transition areas in order to deduce transition classes. This identification allows displaying all classes in a unique map instead of one map per class as in previous modeling. These areas are identified using a thresholding of the trust degrees in the following way: 
1. Let $S$ be a threshold $(S \in[0,1])$

2. For each $V_{i}$ we sort the classes by decreasing order of the trust degrees :

$$
\left\{\mu_{i j_{1}} \geq \mu_{i j_{2}} \geq \cdots \mu_{i j_{n}}\right\} \rightarrow\left\{C_{j_{1}}^{i}, C_{j_{2}}^{i}, \ldots, C_{j_{n}}^{i}\right\}, i \in\{1 \ldots M\}, j_{p} \in\{1 \ldots n\}
$$

3. We affect to each $V_{i}$ the shortest class list such as

$$
E_{i}(S)=\left\{C_{j_{1}}^{i}, C_{j_{2}}^{i}, \ldots, C_{j_{p}}^{i} \mid \sum_{k=1}^{p} \mu_{i j_{k}}>S\right\}
$$

For each $V_{i}: E_{i}(0)=\left\{C_{j_{1}}^{i}\right\}$. So we obtain a unique class for each $V_{i}$ which is equivalent to the generation of a strict classification from a set of $n$ fuzzy classes by choosing the class having the highest trust degree. More generally, if $E_{i}(S)$ is a singleton, there is no ambiguity concerning the class. In the other case, we are confronted to a transition class. Depending on the value of the threshold the number of transition areas varies but the space evolution of these areas is not regular.

By varying $S$ from 0 to 1 we obtain a hierarchy of the transitions. If a transition appears for a low value of $S$ the uncertainty concerning the class affectation is high. If a transition is spatially extended it indicates a slow gradient. The choice of $S$ allows fixing the transition to take into account.

Theoretically every transition between classes is possible and the hierarchy could leads to $2^{\mathrm{n}}$ transitions. But in our case, the spatial coherence of the classes leads to a limited number of transitions.

\section{$4 \quad$ Applications}

Now we are going to apply our approach to a concrete classification of fuzzy sets. We study various types of forests following a progressive evolution (with a gradient covering hundreds of meters).

Indeed, the classification of the forest is based on their floristic composition (species, maturity, etc.) which is a continuous parameter.

As if the granularity of the classes could be discussed the transition aspect of the forests is attested by the experts. We are especially interested in this study to the forest covering the Basse-Terre Island of the Guadeloupe archipelagos.

\subsection{The Reference Map}

We compare our results to a reference map obtained by biologists [16] in 1996. 14 classes $\left\{\mathrm{C}_{1}, \ldots, \mathrm{C}_{14}\right\}$ were identified from the statistical analysis of the floristic composition of 47 observation areas. The map has been manually drawn by the experts taking into account the geographical repartition of the classes and local environmental conditions.

\subsection{Data Used for Supervised Classification}

$V$ is obtained using four information layers about topography (slope, altitude, exposition and hillside) $\left(\mathrm{L}=\left\{L_{1}, L_{2}, L_{3}, L_{4}\right\}\right)$ which generate $417273 V_{i}(M=417273)$. 
These layers give attributes to the $V_{i}$ which will be used during the learning step $\left(F_{i}=\right.$ $\left\{\operatorname{slope}\left(V_{i}\right)\right.$, altitude $\left(V_{i}\right)$, exposition $\left(V_{i}\right)$, hillside $\left.\left.\left(V_{i}\right)\right\}\right)$.

We use the class defined by the experts. The learning set $(A)$ is obtained by selecting the $V_{i}$ containing the 47 observations.

\subsection{Strict Classification $(S=0)$}

In order to compare our approach with the existing classification we apply the algorithm with $S=0$. This naturally leads to a classification without transition classes.

Figure 2 shows two extracts of the National Park of Guadeloupe obtained with FT and C4.5 decision trees. A first visual analysis shows coherence between the two results and also between these results and the reference map.

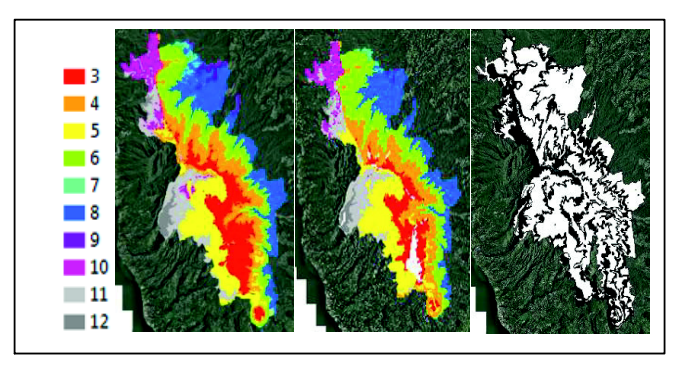

C4.5

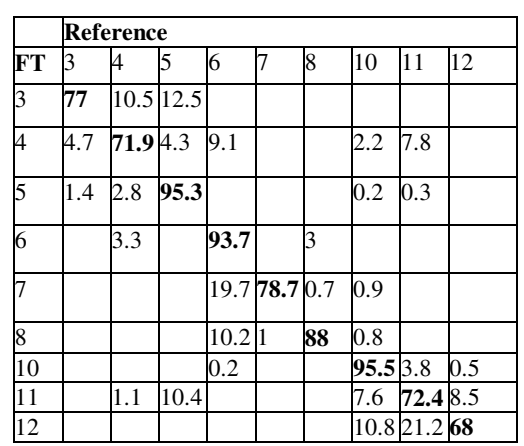

FT-Ref. Confusion matrix

Fig. 2. Automatic classification extracts and differences

Indeed the map in the middle of Figure 2 presents the differences (black) between the reference map and FT classification. We observe that the two classifications are globally identical (few differences). Conflicts areas are localized at the border of the classes. Some borders are exactly localized at the same place and others have more extended differences.

The confusion matrix gives a dominant diagonal with a good classification rate from $68 \%$ to $95.5 \%$ (average $82.3 \%$ ). Regarding that the reference classification has been made manually we can validate the algorithm and the obtained classifications.

\subsection{Fuzzy Classification and Representation}

We give now an illustration of the fuzzy maps and their simplified views $m_{k}=\left\{\mu_{l k}\right.$, $\left.\ldots, \mu_{M k}\right\}$. Figure 3 shows the $m_{9}$ raw and simplified maps. The simplified map in the middle is based on 5 intervals $\left(g_{v}=5\right)$ centered on the more represented values of the trust degree $\left(\left|G_{k p}^{v}\right| \simeq \frac{M}{5}\right) I=\{0,0.03,0.07,0.13,0.22,1\}$, and in the right part with 10 intervals $\left(g_{v}=10\right)$ centered on the highest values $I=\{0,0.14,0.5,0.65,0.73,0.80$, $0.86,0.91,0.95,0.98,1\}$. This last map is the most interesting for our application. We observe the non regular aspect of the different areas compared to regular buffers presents in the literature. 


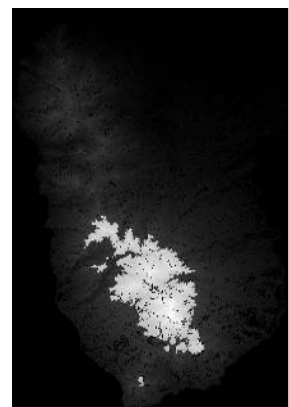

$m_{9}$

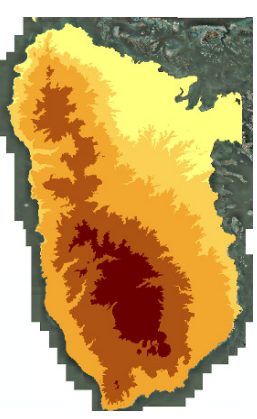

$v_{l}, m_{9}$

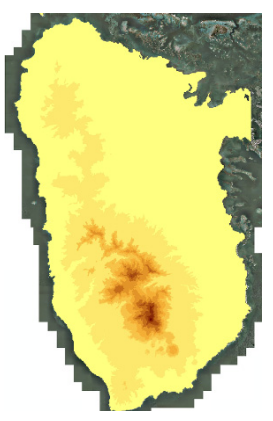

$v_{2}, m_{9}$

Fig. 3. Fuzzy vectorial map (raw : left, simplified : middle and right)

\subsection{Transition Classes}

We now illustrate the localization of the transition classes (in black in figure 4) for two threshold values $(S=0.8$ and 0.9$)$. Transition areas are localized at the border of a class. Some transition are very short such as between class 3 (red) and 5 (yellow).

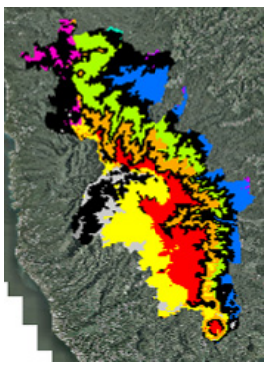

$\mathrm{S}=0.8$

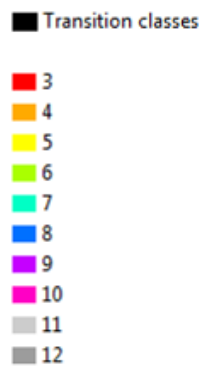

Fig. 4. Transition classes

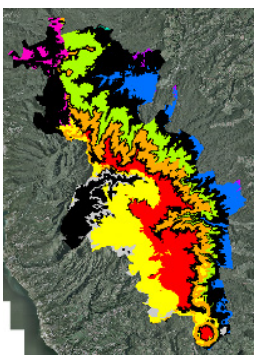

$\mathrm{S}=0.9$

Table 1. Transition hierachy

\begin{tabular}{|c|c|c|}
\hline $\mathrm{S}$ & Transitions & Surface \\
\hline 0 & & 0 \\
\hline 0.1 & $6-7,6-8,7-8$ & $1.2 \%$ \\
\hline 0.3 & $6-7,6-8,7-8, \mathbf{1 0 - 1 2}, \mathbf{1 1 - 1 2}$ & $2.1 \%$ \\
\hline 0.5 & $6-7,6-8, \mathbf{6 - 1 1}, 7-8, \mathbf{8 - 1 1}, \mathbf{1 0 - 1 1}, 10-12,11-12$ & $4.4 \%$ \\
\hline 0.7 & $\begin{array}{c}\text { 3-4, 4-6, 6-7, 6-8, 6-11, 7-8, 8-11, 10-11, 10- } \\
12,11-12\end{array}$ & $21.4 \%$ \\
\hline 0.9 & $\begin{array}{c}3-4,4-6,4-11,6-7,6-8,6-11,7-8,8-11,10-11, \\
10-12,11-12\end{array}$ & $25.1 \%$ \\
\hline 1 & $\begin{array}{c}3-4,4-6,4-11,6-7,6-8,6-11,7-8,8-11,10-11, \\
11-12, \mathbf{6 - 7 - 8}, \mathbf{6 - 8 - 1 1}, \mathbf{1 0 - 1 1 - 1 2}\end{array}$ & $27.2 \%$ \\
\hline
\end{tabular}

Table 1 presents the hierarchy of the transitions obtained by varying $S$ from 0 to 1 . For each value of $S$ the transition set is indicated. We obtain between 3 and 13 transition classes. The first transition that appears is between classes 6,7 and 8 . The 
transition between 6 and 8 grow rapidely with $S$ (also between classes 10 and 11 which appears for $S=0.5$ ). These two transition classes are interesting because they show extended uncertain areas.

\section{$5 \quad$ Perspectives}

The first perspective of the work is the integration of the OKM (Overlap K-Means) algorithm to have an unsupervised approach ([4], [5], [6]). By using a distance in the attributes space, the algorithm allows the affectation of a sample to the union of two or more classes instead of a unique class.

This algorithm could also be applied to the transition areas, after localizing them as described in our approach. The $O K M$ algorithm will allow refining the transition classes.

A second perspective aims to automatically determine the transition classes to take into account by using the importance of the transition (low value of $S$ ) and it's extend.

Then, the localization of the transition areas could guide the choice of the limits of the intervals of the views in order to have well adapted simplified models.

\section{Conclusion}

In a first time, we present in this paper an adaptive fuzzy vector model for spatial data. This model is useful for data having fuzzy borders and well adapted for integration into GIS. The advantage of this structure is its vector representation and its non uniform evolution, in space, instead of regular series of buffers. This takes into account the irregular evolution of the trust degree translating the local environmental conditions.

In a second time, we propose an algorithm allowing, from fuzzy models, the generation of transition classes. The main objective is to identify transition classes when uncertain areas are too spread. The resulting classifications are visually and numerically coherent with the reference classification which allows validating the approach.

\section{References}

1. Altman, D.: Fuzzy set theoretic approaches for handling imprecision in spatial analysis. Internat. J. Geographical Inform. Systems 8(3), 271-289 (1994)

2. Benz, U.C., et al.: Multi-resolution, object-oriented fuzzy analysis of remote sensing data for GIS-ready information. ISPRS Journal of Photogrammetry \& Remote Sensing 5839258 (2004)

3. Burrough, P.A., Frank, A.U.: Geographic Objects with Indeterminate Boundaries, ch. 12, pp. 171-187. Taylor \& Francis, London (1987)

4. Cleuziou, G.: OKM: une extension des k-moyennes pour la recherche de classes recouvrantes. In: 7èmes journées d'Extraction et de Gestion des Connaissances (EGC 2007), pp. 691-702 (2007) 
5. Cleuziou, G.: An extended version of the k-means method for overlapping clustering. In: 19th International Conference on Pattern Recognition (ICPR 2008), pp. 1-4 (2008)

6. Cleuziou, G.: Two Variants of the OKM for Overlapping Clustering. In: Guillet, F., Ritschard, G., Zighed, D.A., Briand, H. (eds.) Advances in Knowledge Discovery and Management. SCI, vol. 292, pp. 149-166. Springer, Heidelberg (2010)

7. Cross, V.V.: Fuzzy extensions for relationships in a generalized object model. International Journal on Intelligent Systems 16, 843-861 (2001)

8. Fisher, P.: Sorites paradox and vague geographies. Fuzzy Sets and Systems 113, 7-18 (2000)

9. Gama, J.: Functional Trees. In: Landwehr, N., Hall, M., Frank, E. (eds.) Logistic Model Trees (2005)

10. Grandchamp, E.: GIS information layer selection directed by remote sensing for ecological unit delineation. In: IGARSS (2009)

11. Grandchamp, E.: Raster-vector cooperation algorithm for GIS. In: GeoProcessing (2010)

12. Kainz: Introduction to Fuzzy Logic and Applications in GIS - Example (2011)

13. Mukhopadhyay, B.: Integrating exploration dataset in GIS using fuzzy inference modeling, GISdevelopment

14. Quinlan, R.: C4.5: Programs for Machine Learning. Morgan Kaufmann Publishers, San Mateo (1993)

15. Raines, G.L., et al.: New fuzzy logic tools in ArcGIS 10. ESRI Communication (2010)

16. Rousteau, A.: Carte écologique de la Guadeloupe. 3 feuilles au 1/75.000ème et notice (36 p.). Conseil Général de la Guadeloupe, Office National des Forêts et Parc National de la Guadeloupe (1996)

17. Sawatzky, D., Raines, G.L., Bonham-Carter, G.: Spatial Data Modeller. Technical Report (2008)

18. Schneider, M.: Spatial Data Types for Database Systems, Finite Resolution Geometry for Geographic Information Systems. LNCS, vol. 1288, 275 p. Springer, Heidelberg (1997)

19. Schneider, M.: Uncertainty Management for Spatial Data in Databases: Fuzzy Spatial Data Types. In: Güting, R.H., Papadias, D., Lochovsky, F.H. (eds.) SSD 1999. LNCS, vol. 1651, pp. 330-351. Springer, Heidelberg (1999)

20. Sunila, R., Horttanainen, P.: Fuzzy Model of Soil Polygons for Managing the Imprecision Interfacing GeoStatistics and GIS (2009)

21. Zhu, A.X., et al.: Soil Mapping Using GIS. Expert Knowledge, and Fuzzy Logic. Simonson, Soil Sci. Soc. Am. J. 65, 1463-1472 (2001) 